\title{
Sphingolipids: agents provocateurs in the pathogenesis of insulin resistance
}

\author{
C. Lipina $\cdot$ H. S. Hundal
}

Received: 29 October 2010 / Accepted: 18 February 2011 /Published online: 6 April 2011

(C) Springer-Verlag 2011

\begin{abstract}
Obesity is a major risk factor for a variety of chronic diseases, including diabetes mellitus, and comorbidities such as cardiovascular disorders. Despite recommended alterations in lifestyle, including physical activity and energy restriction, being the foundation of any antiobesity therapy, this approach has so far proved to be of little success in tackling this major public health concern. Because of this, alternative means of tackling this problem are currently being investigated, including pharmacotherapeutic intervention. Consequently, much attention has been directed towards elucidating the molecular mechanisms underlying the development of insulin resistance. This review discusses some of these potential mechanisms, with particular focus on the involvement of the sphingolipid ceramide. Various factors associated with obesity, such as saturated fatty acids and inflammatory cytokines, promote the synthesis of ceramide and other intermediates. Furthermore, studies performed in cultured cells and in vivo associate these sphingolipids with impaired insulin action. In light of this, we provide an account of the research investigating how pharmacological inhibition or genetic manipulation of enzymes involved in regulating sphingolipid synthesis can attenuate the insulin-desensitising effects of these obesity-related factors. By doing so, we outline potential therapeutic targets that may prove useful in the treatment of metabolic disorders.
\end{abstract}

Keywords Adipose tissue - Caveolae - Ceramide . Gangliosides · GM3 · Skeletal muscle · Sphingomyelin · $\mathrm{PKB} / \mathrm{Akt}$

\section{Lipina $\cdot$ H. S. Hundal $(\square)$}

Division of Cell Signalling and Immunology, Sir James Black Centre,

College of Life Sciences, University of Dundee,

Dundee DD1 5EH, Scotland, UK

e-mail: h.s.hundal@dundee.ac.uk

\author{
Abbreviations \\ aPKC Atypical protein kinase $\mathrm{C}$ \\ AMPK AMP-activated protein kinase \\ CEM Cholesterol-enriched (membrane) microdomain \\ DRM Detergent-resistant (membrane) microdomain \\ GM3 Ganglioside monosialo 3 \\ IMTG Intramuscular triacylglycerol \\ LCFA Long-chain saturated fatty acid \\ $\mathrm{PIP}_{3} \quad$ Phosphatidylinositol $(3,4,5)$-trisphosphate \\ PKB Protein kinase $\mathrm{B}$ \\ PKC Protein kinase $\mathrm{C}$ \\ PP2A Protein phosphatase 2A \\ PTEN Phosphatase and tensin homologue deleted \\ on chromosome ten \\ SPT Serine palmitoyltransferase \\ TLR Toll-like receptor
}

\section{Introduction}

The increasing occurrence of obesity and type 2 diabetes mellitus, attributable to a large extent to sedentary living and excess energy intake, has fuelled a rapidly expanding area of research examining the link between lipid metabolism and the pathogenesis of insulin resistance. Evidence for such a relationship has been acquired from numerous studies involving obese and diabetic humans as well as rodent models exhibiting metabolic dysfunction [1-4]. What has become clear from these investigations is that obesity is a major contributing factor towards the development of the metabolic syndrome and that impairment of insulin action is associated with excess lipid accumulation [3-7]. 
At the molecular level, a key mediator of insulin action is protein kinase B (PKB; also known as Akt), which acts to regulate a number of physiological processes, resulting in an overall hypoglycaemic response [8, 9]. Insulin promotes a reduction in circulating glucose, to a large extent by accelerating its uptake into peripheral tissues such as skeletal muscle and adipose tissue, as well as by stimulating its conversion into energy storage molecules such as glycogen. It is well documented that insulin-stimulated glucose transport into these tissues is significantly attenuated in obese individuals and people with type 2 diabetes [10, 11].

Mechanistically, activation of PKB in response to insulin involves its phosphorylation on two regulatory sites, namely Thr308 in the activation loop and Ser473 in the hydrophobic C-terminal regulatory domain (both sites corresponding to $\mathrm{PKB} \alpha / \mathrm{Akt1})$. Phosphorylation of both residues is required for full activation [12]. This process relies upon the generation of the membrane phospholipid phosphatidylinositol $(3,4,5)$-trisphosphate $\left(\mathrm{PIP}_{3}\right)$ by the enzyme phosphoinositide 3-kinase, resulting in the pleckstrin homology domain-dependent translocation of PKB to the plasma membrane. The high-affinity binding of the Nterminal pleckstrin homology domain of $\mathrm{PKB}$ to $\mathrm{PIP}_{3}$ facilitates a conformational change that allows subsequent phosphorylation at Thr308 by 3-phosphoinositide-dependent protein kinase 1 (PDK1) [13]. The mechanism mediating Ser473 phosphorylation remains controversial although several distinct kinases, including mammalian target of rapamycin complex 2 (mTORC2), have been proposed to be involved, depending on cell type and stimulus [14].

The activation of PKB in response to insulin can be markedly impaired by stimuli such as TNF $\alpha$, glucocorticoids or prolonged exposure to long-chain saturated fatty acids (LCFAs; e.g. palmitate), all of which have been implicated in the development of insulin resistance $[15,16]$. A common feature of these insulin-desensitising agents is their ability to promote the accumulation of sphingolipids such as ceramide [16-18]. Through the use of cell-permeant analogues of ceramide or inhibition of its intracellular synthesis, it is now widely acknowledged that this particular sphingolipid intermediate can regulate a diverse array of cellular processes, including cell survival and apoptosis, inflammatory responses, mitochondrial function and insulin sensitivity [19-21]. It is the last of these, that is, the ability of ceramide to modulate insulin-mediated responses and the potential mechanisms involved, that will be the focus of this review.

\section{Intracellular ceramide synthesis}

Ceramides belong to a group of lipid-derived molecules that consist of a sphingosine base linked to a fatty acid moiety. Although previously thought of as purely structural elements of the membrane lipid bilayer, it is now clear that they also act as important signalling molecules and are implicated in a variety of cellular and physiological processes [22-24]. Ceramides can accumulate in cells via two main routes: the hydrolysis of the membrane phospholipid sphingomyelin, which is coordinated by the enzyme sphingomyelinase [22], or its de novo synthesis from LCFAs such as palmitate, which involves a multi-step biosynthetic pathway, as illustrated in Fig. 1 [22, 23]. A number of different factors, including $\mathrm{TNF} \alpha$, endotoxins and various stress stimuli, have been reported to activate sphingomyelinase leading to the generation of ceramide [22, 25].

The rate-limiting step of de novo synthesis of ceramide is the condensation of a fatty acyl-CoA, usually palmitoylCoA, with serine, which is catalysed by the enzyme serine palmitoyltransferase (SPT), to form 3-ketosphinganine (Fig. 1). The activity of SPT is stimulated in the presence of LCFAs but not unsaturated fatty acids $[16,26]$. The final two steps of the pathway involve the generation of dihydroceramide from sphinganine by the enzyme dihydroceramide synthase and its subsequent conversion into ceramide by dihydroceramide desaturase (Fig. 1). Enhanced ceramide production arising from de novo synthesis has been reported in response to the stimulation of SPT and/or dihydroceramide synthase [27-29]. In addition, ceramide can be further modified into alternative forms, including glucosylceramide and ceramide 1-phosphate, or converted into other metabolites such as sphingosine 1-phosphate (Fig. 1), some of which can induce distinct or even opposite biological effects [22, 30, 31].

\section{The role of ceramide in the pathogenesis of insulin resistance}

So why is ceramide considered to be a contributing factor in the development of insulin resistance? The evidence underpinning this view originates from several important observations. First, sustained exposure of skeletal muscle to non-esterified fatty acids results in the accumulation of intramuscular triacylglycerol (IMTG) and fatty acid-derived molecules such as diacylglycerol and ceramide [3, 5, 19, 29, 32]. However, although the accumulation of IMTG is normally associated with a loss in skeletal muscle insulin sensitivity, the observation that muscle from endurance-trained athletes remains insulin sensitive despite elevated IMTG content (known as the athlete's paradox), initially suggested that increases in intramuscular lipid alone may not be sufficient to explain lipid-induced insulin resistance [7, 33]. Importantly, with respect to this finding, we and others have shown that 


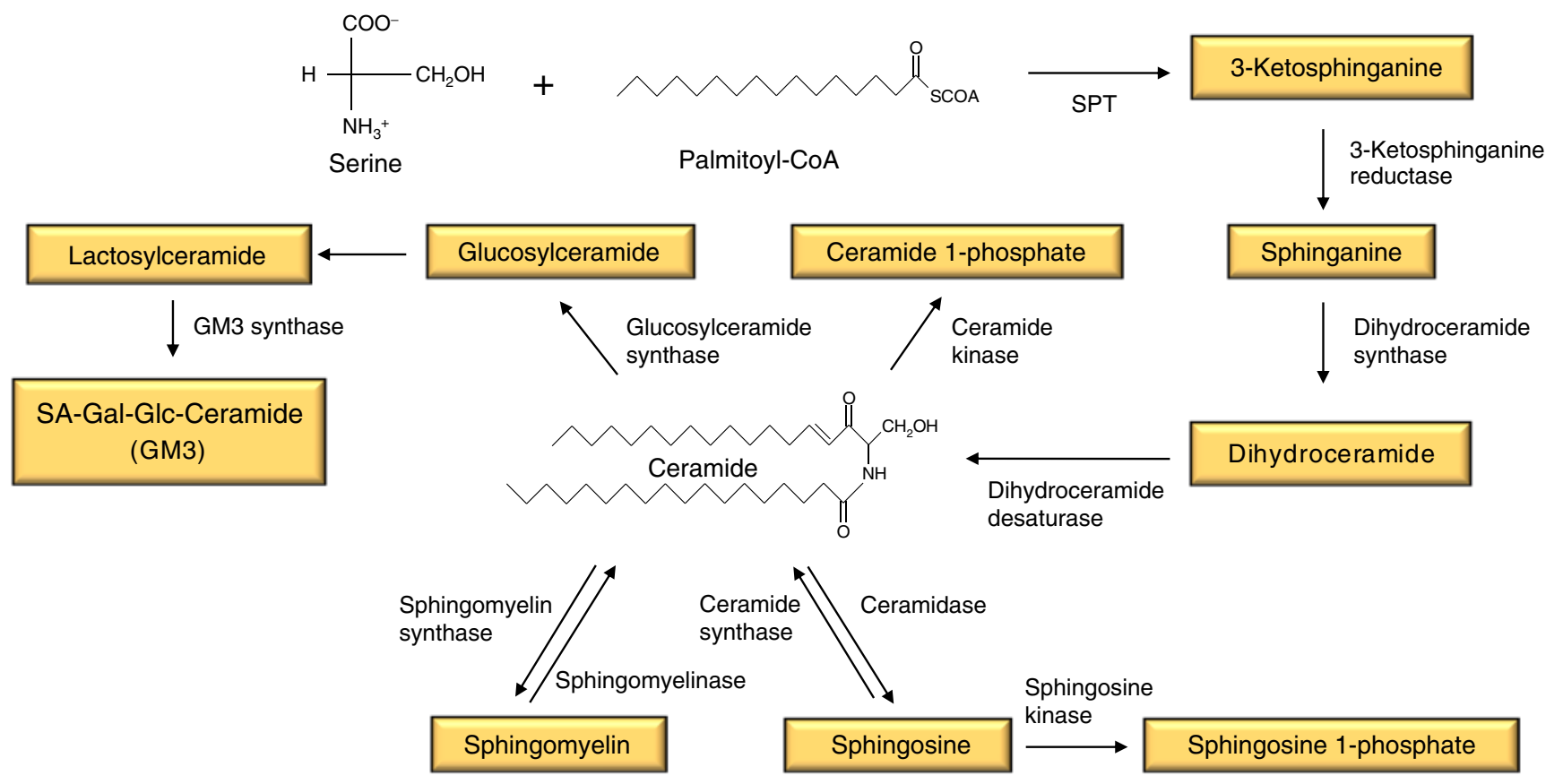

Fig. 1 An overview of the major pathways involved in the synthesis of ceramide and its derivatives. Ceramide can be generated in a number of different ways, including its de novo synthesis involving several key enzymes, such as serine palmitoyl transferase (SPT), or by the hydrolysis of sphingomyelin by the action of sphingomyelinases.

sustained exposure of skeletal muscle to palmitate, the predominant circulating saturated fatty acid, results in markedly impaired insulin-stimulated PKB activation concomitant with inhibition of associated processes such as glucose uptake and glycogen synthesis [8, 34, 35].

Second, given the fact that the de novo synthesis of ceramide can be driven solely by the availability of LCFAs, it has now been demonstrated in a variety of cell lines and in isolated human myoblasts that the insulindesensitising effects of palmitate can also be mimicked by the acute application of ceramide analogues [15, 17-19, $36,37]$. In addition, under those circumstances whereby elevating levels of circulating lipids in rodents either by lipid infusion or high-fat feeding causes insulin resistance, a number of studies have now demonstrated an associated increase in ceramide content within peripheral tissues such as skeletal muscle and adipose tissue [20, 23, 38, 39]. Consistent with this, prolonged incubation of cultured skeletal muscle $\mathrm{C} 2 \mathrm{C} 12$ and L6 myotubes or human myoblasts with palmitate also leads to an increase in ceramide content in accordance with impaired insulinstimulated PKB activity [17, 19, 29, 37, 40]. With regards to human physiology, elevations in skeletal muscle ceramide and/or plasma ceramide levels have been reported in obese insulin-resistant and diabetic individuals, as well as in response to lipid infusion [20, 40, 41]. Furthermore, intramuscular ceramide accumulation is also known to
Both these processes, which take place at the plasma membrane, can be induced in response to various stimuli (as discussed in the text). The modification of ceramide (e.g. by its phosphorylation or glucosylation) produces molecular species with similar or distinct biological activities. Gal, galactose; Glc, glucose; SA, sialic acid

occur in certain animal models of insulin resistance, including Zucker ( $f a / f a)$ rats [42, 43].

Third, in line with being a measure of obesity prevention, the participation of insulin-resistant rodents and humans in prolonged exercise regimens has been shown to markedly improve insulin sensitivity in association with reduced intramuscular ceramide [7, 20, 44]. Finally, and perhaps most importantly, the inhibition of ceramide synthesis can counteract the antagonistic effects of saturated fatty acids on insulin-regulated PKB signalling (discussed below) [15, 17, 20]. Collectively, these observations strongly suggest that ceramide plays a key role in the development of insulin resistance, primarily through its ability to inhibit PKB [17, 19, 34].

Since initial work demonstrated that ceramide does not directly interact with $\mathrm{PKB}$ or inhibit upstream signalling events such as the activation of phosphoinositide 3-kinase by hormonal stimuli [19, 20, 45], researchers undertook alternative approaches in an attempt to explain its suppressive effect. Studies performed over the past decade have identified two distinct mechanisms that may be involved. The evidential basis of these proposed mechanisms, as well as the potential involvement of ceramide-rich membrane lipid rafts known as caveolae, will be discussed in the following sections. It must be noted, however, that although the mechanisms are treated separately (as depicted in Fig. 2b), their occurrence may not necessarily be mutually exclusive. 


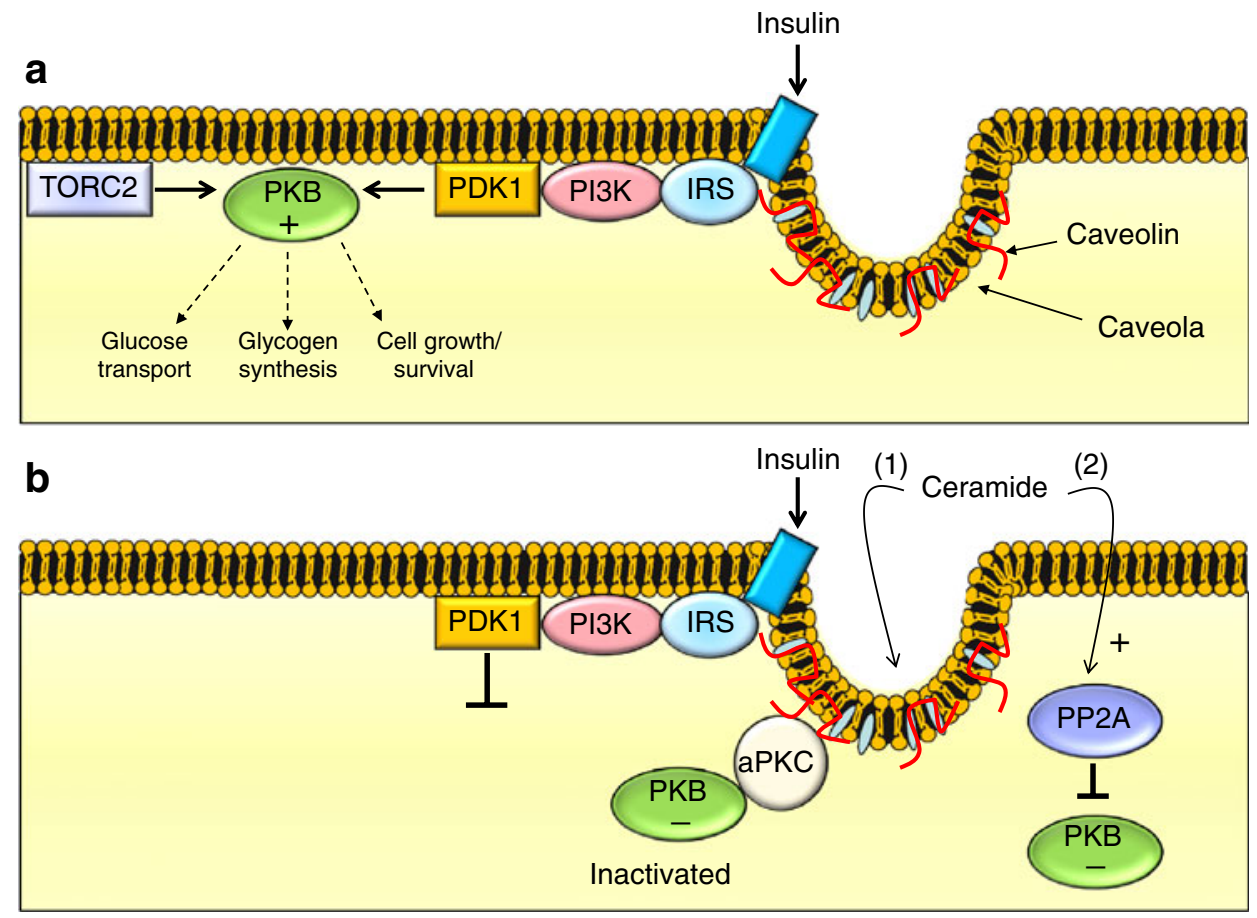

Fig. 2 Proposed mechanisms by which ceramide inhibits PKB. Normally, insulin binds to its receptor at the plasma membrane promoting the activation of $\mathrm{PKB}$ through the tyrosine phosphorylation of IRS proteins and the subsequent activation of phosphoinositide 3kinase (PI3K). The associated increase in $\mathrm{PIP}_{3}$ levels leads to the recruitment of $\mathrm{PKB}$ to the plasma membrane, where it is activated by the upstream kinases 3-phosphoinositide-dependent protein kinase 1 (PDK1) and TORC2. a The activation of PKB results in the stimulation of insulin-regulated processes such as glucose transport and glycogen synthesis. $\mathbf{b}$ In the presence of increased intracellular levels of ceramide, the ability of insulin to stimulate PKB is impaired

\section{Involvement of phosphatase activity in ceramide-mediated inhibition of $\mathrm{PKB}$}

Initial evidence implicating a role for protein phosphatases in mediating the inhibitory effects of ceramide on insulinstimulated PKB activity was obtained from studies performed using $\mathrm{C} 2 \mathrm{C} 12$ myotubes and brown adipocytes treated with the protein phosphatase inhibitor okadaic acid $[18,46]$. Although okadaic acid does not selectively target one particular protein phosphatase isoform (i.e. inhibits protein phosphatases 1 [PP1] and 2A [PP2A]), it has subsequently been demonstrated, through use of small $\mathrm{t}$ antigen (which displaces regulatory subunits of PP2A), that this inhibitor can prevent ceramide-mediated inhibition of PKB by specifically inhibiting PP2A, a serine/threonine phosphatase implicated in the dephosphorylation of PKB [20]. To support this, ceramide has been shown to act as a positive allosteric activator of PP2A [47, 48]. Therefore, one way that ceramide can act to inhibit PKB activity is by promoting its dephosphorylation at Thr308 and Ser473 through activation of PP2A (see Fig. 2b). However, in certain cell types, such as L6 myotubes and white adipose, by one of two established mechanisms. In the first scenario (1), the increase in caveolar ceramide content results in the activation of $\mathrm{aPKC} \lambda / \zeta$ and promotes the association of $\mathrm{aPKC} \lambda / \zeta$ and $\mathrm{PKB}$ within these membrane microdomains. As a result of this interaction, PKB remains in a repressed state and is unable to transduce signalling to downstream components. Alternatively (2), the intracellular ceramide generated leads to the direct activation of PP2A, causing the dephosphorylation and inactivation of PKB. In both cases (1 and 2), the inhibition of PKB is associated with the impairment of key insulinregulated responses

the ceramide-mediated inhibition of PKB cannot be antagonised by co-treatment with okadaic acid alone, thereby suggesting the involvement of an alternative mechanism(s) [19, 34].

\section{The role of atypical protein kinase $\mathrm{C}$ isoforms in ceramide-induced insulin resistance}

The second way that ceramide is known to promote insulin resistance involves the disruption in membrane translocation and reduced phosphorylation of PKB by a process dependent on the activation of atypical protein kinase $\mathrm{C}$ (aPKC) $\lambda / \zeta$ isoforms. A reciprocal relationship between PKC activity and insulin action was initially established in human muscle strips that were incubated in the presence of pharmacological PKC activators or inhibitors [49]. From this study and others, it has emerged that whilst activation of $\mathrm{aPKC} \lambda / \zeta$ may be involved in suppressing insulin-stimulated PKB activity and associated processes, inhibition of aPKCs is able to promote an insulin-sensitising effect [49-52]. In addition, pharmacological inhibition of PKC 
activity has also been shown to prevent the ceramide-induced loss of insulin-stimulated PKB activation in both isolated human adipocytes and L6 myotubes $[51,53]$.

To investigate how ceramide blocks the translocation of PKB to the plasma membrane, initial work by Summers' group demonstrated that whilst ceramide analogues prevent the membrane localisation of the pleckstrin homology domain of PKB, they do not alter the accumulation of 3'phosphoinositides [45]. This therefore suggested that the inhibitory effect of ceramide occurs independently of 3'phosphoinositide production.

Subsequent studies demonstrated that ceramide can directly activate aPKC $\lambda / \zeta$ isoforms in vitro by binding to the putative ceramide-binding region of the protein, also known as the cysteine-rich domain [50]. Importantly, aPKC $\lambda / \zeta$ also interacts with and suppresses the activity of PKB [50, 51, 54]. Follow-up work from our group established that the interaction between $\mathrm{PKC} \lambda / \zeta$ and $\mathrm{PKB}$ requires the PKB pleckstrin homology domain, and that ceramide-activated $\mathrm{PKC} \lambda / \zeta$ phosphorylates a threonine or serine residue at site 34 (dependent on PKB isoform) within this region [51]. As a result, reduced affinity of the pleckstrin homology domain towards $\mathrm{PIP}_{3}$ prevents the recruitment of PKB to the plasma membrane and its subsequent activation. Based on these observations it has been proposed that increases in intracellular ceramide leading to the activation of aPKC $\lambda / \zeta$ promotes the stabilisation of the aPKC $\lambda / \zeta-\mathrm{PKB}$ complex and attenuates the recruitment of PKB to the plasma membrane as a result of disrupted $\mathrm{PIP}_{3}$ binding (Fig. 2b) [51, 53].

\section{Influence of caveolae in mediating the effects of ceramide}

It is now widely acknowledged that the plasma membrane is not a homogenous structure but instead consists of microdomains (or rafts), each with their own characteristic lipid composition [55]. Indeed, lipid-derived molecules such as cholesterol or sphingolipids can segregate into such microdomains, which are more ordered and less fluid in nature compared with the rest of the plasma membrane [56]. Detergent treatment of cell membranes allows the isolation of detergent-resistant microdomains (DRMs) that are enriched in sphingolipids, including ceramide [57]. This particular subset of lipid rafts, termed cholesterol-enriched membrane microdomains (CEMs), are characterised by the presence of integral membrane proteins termed caveolins (namely, caveolin-1, -2, -3 and -4) [57].

The caveolins themselves are cholesterol-binding proteins that associate to form the flask-like invaginations that characterise caveolae and, importantly, are capable of acting as scaffolding proteins onto which signalling complexes assemble. For this reason, CEMs play an important role in numerous signalling pathways, including insulin-mediated responses [57, 58]. Our previous work has demonstrated that acute ceramide treatment (or prolonged incubation with palmitate) promotes the recruitment of both $\mathrm{aPKC} \lambda / \zeta$ and PKB to these CEMs [53]. By doing so, aPKC $\lambda / \zeta$ would not only be exposed to a ceramide-enriched environment, rendering it active, but would simultaneously be colocalised with PKB, thereby suppressing its activity.

Intriguingly, disrupting these microdomains using a cholesterol-depleting agent prevents the recruitment of these kinases to DRMs and ameliorates ceramide-mediated loss in PKB activity [53]. Although a direct physical association between aPKC $\lambda / \zeta$ and PKB has yet to be established within CEMs, ceramide has been shown to promote both aPKC $\lambda / \zeta_{-}$ PKB and caveolin- $1-\mathrm{aPKC} \lambda / \zeta$ interactions in 3T3-L1 adipocytes, suggesting that caveolins may act to bridge the association between $\mathrm{PPKC} \lambda / \zeta$ and PKB [53]. This may be at least partly due to regions within caveolin proteins, known as caveolin scaffolding domains, that allow them to interact directly with aPKC $\lambda / \zeta[59]$.

In addition to promoting the recruitment of PKB and aPKC $\lambda / \zeta$, ceramide exposure can also significantly elevate levels of phosphatase and tensin homologue deleted on chromosome ten (PTEN) in caveolin-enriched DRM fractions $[53,60]$. The tumour suppressor PTEN acts as a phosphoinositide phosphatase and its increased abundance in CEMs would likely result in reduced localised levels of 3'-phosphoinositides. Together with the presence of activated aPKC $\lambda / \zeta$, the CEMs create a highly repressive environment for PKB under such circumstances. Furthermore, the fact that ceramide does not suppress total insulinstimulated production of $3^{\prime}$-phosphoinositides at the plasma membrane further supports the idea that this repressive environment is unlikely to be extended to the bulk planar membrane and is rather more focused in nature [51].

Intriguingly, a recent study by Blouin et al. demonstrated that the mechanism by which ceramide inhibits PKB may itself depend on cellular CEM abundance [48]. In cells that are abundant in caveolae, such as differentiated 3T3-L1 adipocytes, ceramide predominantly acts to inhibit PKB by recruiting aPKC $\lambda / \zeta$ and PKB to these membrane domains. In contrast, in CEM-deficient 3T3-L1 preadipocytes, a mechanism dependent on PP2A activity occurs, and this can be prevented by cotreatment with okadaic acid [48]. Furthermore, it has been shown that ceramide-activated PP2A is able to dephosphorylate a cytosolic insulin-stimulated PKB construct lacking its pleckstrin homology domain, thereby excluding a role for the membrane translocation of PKB in this process [20]. To complement these findings, the study by Blouin et al. also demonstrated that the ceramide-activated PP2A pathway is found to dominate in fibroblasts with a low 
CEM abundance but switches to a aPKC $\lambda / \zeta$-dependent pathway following overproduction of caveolin-1 [48]. Although the mechanism responsible for promoting this switch is not fully understood, work performed in prostate cancer cells determined that caveolin-1 can interact with and suppress PP2A [61]. This observation suggests that disruption of caveolae or suppressed caveolin abundance may cause the 'release' of PP2A into an unbound state in the cytosol where it would be more susceptible to activation by ceramide.

Despite the implication of caveolins and caveolae in the development of insulin resistance, the reader must also bear in mind the important role that these membrane proteins play in maintaining proper signal transduction. For example, because caveolins act as scaffolding proteins involved in organising the assembly of signalling complexes, it comes as no surprise that caveolin deficiency causes impaired insulin responses as well as a wide range of phenotypes and disorders [62]. In addition, a recent study has shown that caveolins may also confer a protective effect against elevated fatty acid levels [63]. Consequently, the role of caveolins and caveolae in insulin signalling can be viewed in two different ways-either as an aid to preserving insulin-regulated processes or as membrane microstructures, which, under certain circumstances, may contribute to the development of impaired insulin action.

\section{The potential of modulating sphingolipid/ ceramide-associated signalling as a therapeutic intervention}

(1) Inhibition of SPT activity Work investigating the mechanisms involved in ceramide-induced insulin resistance has led to a number of potential therapeutic targets being proposed to tackle obesity-related diseases. However, it would be reasonable to speculate that preventing ceramide accumulation in response to elevated levels of circulating lipids or other stimuli that promote insulin resistance may be beneficial in ameliorating any insulindesensitising effects. With this in mind, the most commonly studied molecular target involved in suppressing ceramide production is the enzyme SPT which, as mentioned above, catalyses the initial rate-limiting step in de novo ceramide synthesis (Fig. 1) [64]. Several potent inhibitors of SPT have been documented, although the most widely used is myriocin, a naturally occurring fungal metabolite isolated from Myriococcum albomyces [64]. Studies performed by our group and others have demonstrated that acute inhibition of SPT using myriocin ameliorates the loss in insulin-stimulated PKB activation in cultured L6 or C2C12 myotubes caused by palmitate-driven ceramide synthesis $[15,29,35,37]$. With regards to equivalent studies carried out in vivo, administration of myriocin is also found to attenuate PKB inhibition in response to lipid infusion or high-fat feeding, as well as improving glucose tolerance and peripheral insulin sensitivity in obese $o b / o b$ mice and Zucker Diabetic Fatty rats $[16,23,65]$. As expected, these beneficial effects of myriocin are associated with reduced levels of circulating ceramide and are reproduced when alternative inhibitors of de novo ceramide synthesis such as L-cycloserine (which also inhibits SPT) and fumonisin B1 (dihydroceramide synthase inhibitor) are used [15, 16, 35].

Surprisingly, however, a recent study demonstrated that, in contrast to acute inhibition of SPT, its chronic suppression in L6 myotubes by pharmacological means or shorthairpin RNA-mediated silencing, fails to prevent the insulin-desensitising effects of palmitate [29]. Although initially unexpected, a possible explanation for this apparent lack of effect may involve the channelling of palmitate into other lipid intermediates that can contribute towards insulin resistance. Consistent with this idea, the same study was able to show a comparable significant increase in diacylglycerol content, which was coupled with a loss in insulin sensitivity, in palmitate-treated L6 cells containing a stable loss of SPT expression and in L6 cells treated over a longer period (7 days) with myriocin. Intracellular diacylglycerol accumulation itself has been linked to the activation of novel and conventional PKCs (such as PKC $\theta$ ) that are implicated in lipid-induced insulin resistance [66]. Although the full spectrum of serine sites that may be targeted by different PKC isoforms on IRS proteins remains poorly defined, $\mathrm{PKC} \theta$ has been suggested to directly promote phosphorylation of Ser1101 of IRS-1, resulting in reduced insulin sensitivity [67]. Therefore, in this case a PKC-mediated increase in IRS-1 or insulin receptor serine phosphorylation may underlie palmitate-induced insulin resistance under conditions where SPT expression/activity becomes chronically suppressed [29].

Importantly, based on the observations made in this particular study, although inhibiting SPT with pharmacological inhibitors can improve insulin sensitivity, at least in the short term, any apparent long-term benefits would need to be carefully assessed in light of the possible accumulation of other fatty acid-derived metabolites with the potential to induce insulin resistance. The fact that these observations regarding the sustained inhibition of SPT activity are not fully consistent with studies performed in vivo, the possibility of greater efficacy of SPT inhibitors in tissues other than skeletal muscle cannot be discounted and requires further investigation. In addition, there is the possibility that myriocin treatment may act to simultaneously reduce levels of other sphingolipids derived from ceramide (e.g. glycosphingolipids), thereby contributing to its beneficial effects. However, such consequential responses of myriocin administration have not yet been reported. Furthermore, although such studies commonly 
support a role for SPT in modulating insulin sensitivity, its effect on the regulation of body weight and energy expenditure still remain unclear based on discrepancies presented in literature [16, 23, 65].

As well as targeting SPT directly, there is also evidence to suggest that manipulating the activity of molecular targets or pathways that do not directly participate in the de novo synthesis of ceramide, may also result in the modulation of SPT activity and/or ceramide production. For example, overproduction of stearoyl-CoA desaturase, an enzyme involved in the conversion of saturated fatty acids into monounsaturated fatty acids in L6 myotubes leads to both improved insulin sensitivity and the attenuation of ceramide accumulation in response to palmitate exposure [68]. Furthermore, it has recently been demonstrated that adiponectin, an insulin-sensitising adipocyte-derived secretory factor, can act through its receptors (AdipoR1 and AdipoR2) to lower ceramide levels by stimulating the activity of ceramidase, an enzyme that catalyses the degradation of ceramide [69].

It is widely acknowledged that certain exercise regimens can alleviate diet-induced obesity and insulin resistance. Indeed, a number of independent studies have demonstrated that exercise training is associated with suppression of circulating and/or tissue ceramide content [33, 44, 70]. For example, in a study by Bruce et al., obese humans who participated in 8 weeks of exercise training showed a decrease of approximately $40 \%$ in skeletal muscle ceramide content and an associated improvement in insulin sensitivity [44]. One potential contributing factor to exerciseinduced suppression of ceramide generation may involve the activation of AMP-activated protein kinase (AMPK) [71]. AMPK activation by pharmacological means has been shown to prevent palmitate-induced SPT activity [72], thereby suggesting the possibility of a similar response to exercise-stimulated AMPK in vivo [73]. Furthermore, the activation of AMPK, which has a stimulatory effect on mitochondrial $\beta$-oxidation of fatty acids, acts to shunt palmitate away from ceramide synthesis towards oxidation $[44,74,75]$. Indeed, accelerating the rate of fatty acid import into and its subsequent oxidation in mitochondria can alleviate high-fat diet-induced insulin resistance [76]. Consistent with this idea, a recent study showed that preincubating $\mathrm{C} 2 \mathrm{C} 12$ myotubes with the monounsaturated fatty acid oleate or introducing a mutant active form of carnitine palmitoyltransferase 1 (a key positive regulator of LCFA $\beta$-oxidation), can prevent palmitate-induced ceramide accumulation and insulin resistance by increased channelling of the saturated fatty acid towards triacylglycerol and/or through its enhanced oxidation [75]. This therefore raises the possibility that glucose-lowering pharmacological activators of AMPK (or exercise mimetics) and/or consumption of diets with a higher proportion of unsaturated fatty acids, may act to prevent diet-induced obesity and impaired metabolic function, at least in part by restricting ceramide generation.

(2) Inhibition of sphingomyelinase As well as through de novo synthesis, ceramide can also be produced by the action of sphingomyelinase enzymes that are activated in response to stimuli such as TNF $\alpha$ [77]. Sphingomyelinase generates ceramide through the hydrolysis of sphingomyelin (Fig. 1). Interestingly, the abundance and/or activity of either neutral or acid sphingomyelinases has been reported to be elevated in the adipose tissue of $o b / o b$ and high-fat diet-induced obese mice as well as in liver in response to high-fat feeding [38, 78, 79]. Furthermore, a recent study demonstrated that exposure of $\mathrm{C} 2 \mathrm{C} 12$ myotubes to exogenous sphingomyelinase can elevate intracellular ceramide levels; however, effects on insulin sensitivity were not determined in this particular study [80].

It is therefore plausible that increased levels and/or activity of sphingomyelinase-stimulating stimuli or secreted serum sphingomyelinase may also contribute to ceramide accumulation in peripheral tissues $[80,81]$. Indeed, it has been shown that genetic loss of acid sphingomyelinase can prevent diet-induced obesity, hyperglycaemia and insulin resistance in mice lacking the LDL receptor $\left(\mathrm{Ldll}^{--}\right)$, which are prone to metabolic disease when placed on highfat diets [82]. Furthermore, a recent study by Bioni et al. demonstrated that treatment of mice with the acidic sphingomyelinase inhibitor amitriptyline attenuates highfat diet-induced elevations in plasma ceramide and improves insulin sensitivity [78].

An alternative mechanism by which saturated fatty acids may lead to the activation of sphingomyelinase could involve Toll-like receptor (TLR) signalling. Toll-like receptors are a family of proteins that play an important role in the innate immune system [83]. Although normally activated in response to pathogens and cell stress, it is now apparent that saturated fatty acids may themselves act as ligands for two TLR isoforms in particular, namely TLR2 and TLR4, both of which have been linked to the development of obesity and insulin resistance [84, 85]. Intriguingly, activation of TLR2/4-mediated signalling by lipopolysaccharide, an endotoxin isolated from Gramnegative bacteria, has been shown to promote the accumulation of ceramide [86]. Therefore, together with its involvement in the production of cytokines such as TNF $\alpha$ and IL-6, regulation of TLR2/4-mediated signalling may play an additional role in modulating ceramide generation.

To summarise, although studies determining the effects of sphingomyelinase activity on insulin sensitivity and metabolic efficiency are still comparatively few in number, the findings from them so far hold promise that sphingomyelinase enzymes may play a significant role in the 
pathogenesis of type 2 diabetes and are therefore a potential therapeutic target.

(3) The ganglioside GM3 as a potential mediator of insulin resistance Another class of ceramide-derived sphingolipids that have been implicated as modulators of transmembrane signalling are the gangliosides. Gangliosides are complex sialic acid-containing glycosphingolipids consisting of a ceramide moiety linked to an oligosaccharide chain [87]. One of the gangliosides in particular, GM3 (ganglioside monosialo 3), has been shown to modulate insulinmediated signalling. The synthesis of GM3 involves the glucosylation of ceramide (see Fig. 1), and important enzymes in this process include glucosylceramide synthase, which catalyses the initial glycosylation step, and GM3 synthase, an intracellular sialyltransferase that promotes the conversion of lactosylceramide to GM3 (Fig. 1).

Data from several studies now suggest that GM3 may be involved in mediating insulin-desensitising effects and, in particular, that of pro-inflammatory cytokines such as $\mathrm{TNF} \alpha[26,88,89]$. First, insulin resistance induced by $\mathrm{TNF} \alpha$ in $3 \mathrm{~T} 3-\mathrm{L} 1$ adipocytes has been associated with elevated GM3 levels caused by increased GM3 synthase abundance and activity [88]. Second, pharmacological inhibitors of glucosylceramide synthase, which deplete cellular GM3, can prevent the inhibitory effects of TNF $\alpha$ on insulin signalling in cultured 3T3-L1 adipocytes [88, 90]. Furthermore, GM3 itself can mimic the effects of $\mathrm{TNF} \alpha$ by inhibiting tyrosine phosphorylation of the insulin receptor and IRS-1, as well as suppressing insulinstimulated glucose uptake [89, 91]. Concomitantly, improved tyrosine phosphorylation of the insulin receptor has been reported in response to pharmacological inhibition of glucosylceramide synthase and in GM3 synthase-deficient mice $[90,92]$. Furthermore, studies by Kabayama et al. demonstrated that GM3 can directly interact with the insulin receptor and by doing so, causes its displacement from caveolae leading to attenuated insulin signalling [93]. This observation implies that GM3 may regulate insulin signalling via its effects on insulin receptor membrane localisation and activity $[89,93]$.

Further supporting a role for GM3 in the development of insulin resistance in vivo, administration of glucosylceramide synthase inhibitors [ $N$-(5-adamantane-1-yl-methoxypentyl)-deoxynojirimycin (AMP-DNM) and Genz-123346] has been shown to improve both glucose tolerance and insulin sensitivity in skeletal muscle and liver of $o b / o b$ mice and Zucker diabetic rats, as well as in diet-induced obese mice, without any significant alteration in food intake or loss of body weight $[90,92]$. Similar effects are observed in mice lacking GM3 (due to GM3 synthase deficiency), which display protection against high-fat diet-induced insulin resistance [91]. Interestingly, insulin sensitivity is found to be improved in mice with elevated production of the GM3 degrading sialidase Neu3 in liver [94]. However, to our knowledge there are no data showing whether changes in Neu3 abundance and/or activity is regulated by obesity.

To date, one pilot study has reported increased circulating levels of GM3 in obese type 2 diabetic individuals as well as in individuals displaying hyperglycaemia and/or hyperlipidaemia [95]. This is in agreement with the elevated GM3 levels present in skeletal muscle from certain obese insulin resistant murine models, although as yet there

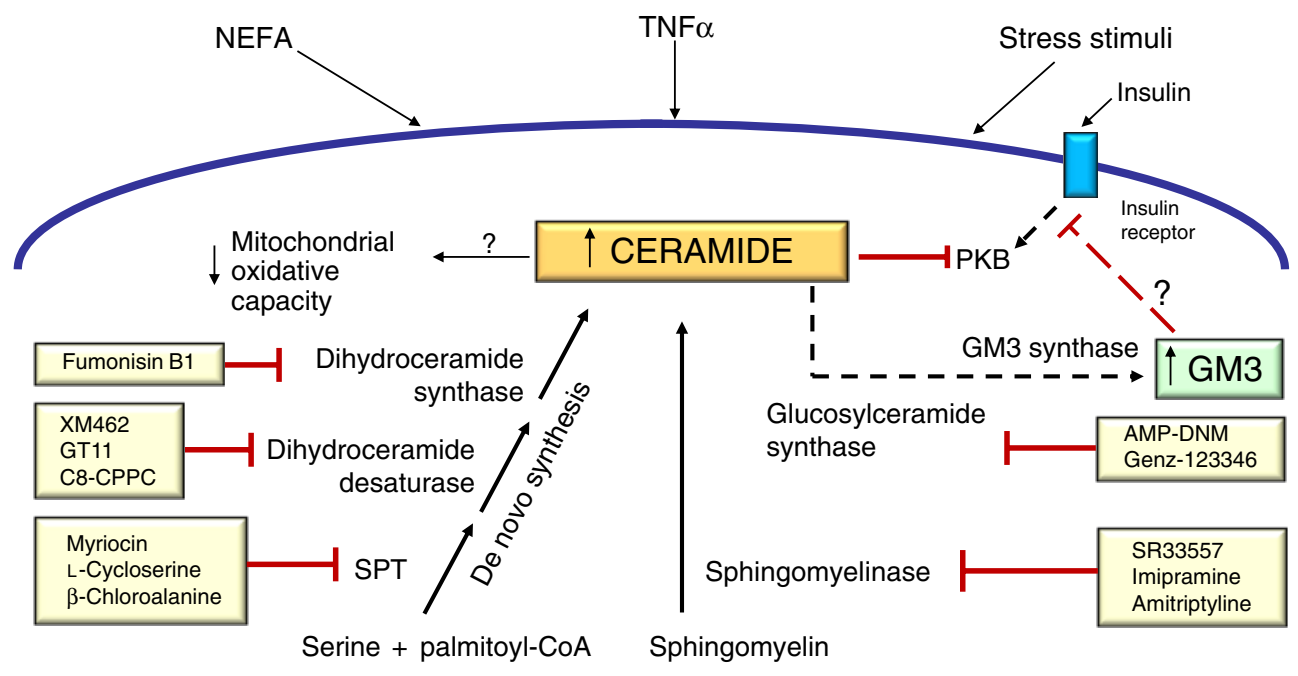

Fig. 3 Schematic representation of potential targets for glucoselowering therapies involved in sphingolipid metabolism. Intracellular ceramide can accumulate in response to various cellular stimuli via two main routes: de novo synthesis or hydrolysis of sphingomyelin. Potential therapeutic targets for the treatment and/or prevention of

obesity-related disorders that are involved in the synthesis of ceramide and the ganglioside GM3 are presented, including the identities of some known pharmacological inhibitors used to regulate the different components. C8-CPPC, C8 cyclopropenylceramide 
is no supporting evidence in tissues obtained from obese or diabetic humans [88]. Intriguingly, individuals with type I Gaucher's disease, a lysosomal storage disorder caused by an impairment in glucosylceramide breakdown leading to elevated levels of GM3, are also found to exhibit insulin resistance [87]. Collectively, these observations open up the possibility that GM3 may serve as a diagnostic marker for metabolic-related disorders, and that therapeutic interventions aimed at reducing GM3 levels in vivo may prove to be a useful strategy for combating insulin resistance.

\section{Summary and concluding remarks}

In summary, a number of studies have demonstrated an inverse relationship between ceramide content and insulin sensitivity. However, as work in our lab and others has demonstrated, ceramide should be viewed as one of multiple factors involved in impaired insulin action. The reader must also be aware that, although the pharmacological and genetic evidence presented in this review strongly implicates ceramide or its derivatives in the development of insulin resistance, some counter-evidence exists to suggest that it may not be the key or sole intermediate involved. For example, certain studies have reported no significant differences in human muscle ceramide content in individuals with varying insulin sensitivity or adiposity [3, $96,97]$. Also, lipid infusion in rodents (depending on lipid composition) can promote insulin resistance without a significant change in tissue ceramide content $[96,98]$. In addition, some animal models displaying marked elevations in hepatic ceramide content do not show a corresponding impairment of insulin-mediated signalling in the liver $[6,99]$.

However, despite the lack of dramatic changes in total ceramide levels, one cannot rule out the possibility that alterations in the type of ceramide species present may also be an important factor in determining insulin sensitivity. Crucially, it must be noted that the methods used to determine ceramide content in these studies do not discriminate between ceramide and biologically inactive dihydroceramide. Therefore, significant differences in the levels of the inactive analogue may have a considerable effect on any results obtained. Another major issue is the relatively small sample sizes employed in the studies performed to date.

It is also possible that ceramide may affect whole body metabolism in other ways. For example, elevated levels of ceramide, which have been shown to promote mitochondrial dysfunction, could if sustained over a period of time, lead to reduced oxidative capacity in metabolically active tissues such as skeletal muscle [21]. In either case, if ceramide does play a crucial role in the pathogenesis of the metabolic syndrome, the fact that ceramide (and its derivatives) can be generated in a number of different ways suggests that, as outlined in Fig. 3, targeting multiple pathways may be the most effective strategy for the treatment of metabolic disorders and associated complications [78, 80, 100].

Acknowledgements Research in the authors' laboratory has been supported by European Commission (contract LSHM-CT-20004005272), Biotechnology and Biological Sciences Research Council (BBSRC), AstraZeneca, Diabetes Research \& Wellness Foundation and Diabetes UK.

Duality of interest The authors declare that there is no duality of interest associated with this manuscript.

\section{References}

1. Dohm GL, Tapscott EB, Pories WJ et al (1988) An in vitro human muscle preparation suitable for metabolic studies. Decreased insulin stimulation of glucose transport in muscle from morbidly obese and diabetic subjects. J Clin Invest 82:486-494

2. Greco AV, Mingrone G, Giancaterini A et al (2002) Insulin resistance in morbid obesity: reversal with intramyocellular fat depletion. Diabetes 51:144-151

3. Itani SI, Ruderman NB, Schmieder F, Boden G (2002) Lipidinduced insulin resistance in human muscle is associated with changes in diacylglycerol, protein kinase $\mathrm{C}$, and $\mathrm{I} \kappa \mathrm{B}-\alpha$. Diabetes 51:2005-2011

4. Muoio DM (2010) Intramuscular triacylglycerol and insulin resistance: guilty as charged or wrongly accused? Biochim Biophys Acta 1801:281-288

5. Goodpaster BH, Kelley DE (2002) Skeletal muscle triglyceride: marker or mediator of obesity-induced insulin resistance in type 2 diabetes mellitus? Curr Diab Rep 2:216-222

6. Monetti M, Levin MC, Watt MJ et al (2007) Dissociation of hepatic steatosis and insulin resistance in mice overexpressing DGAT in the liver. Cell Metab 6:69-78

7. Dube JJ, Amati F, Stefanovic-Racic M, Toledo FG, Sauers SE, Goodpaster BH (2008) Exercise-induced alterations in intramyocellular lipids and insulin resistance: the athlete's paradox revisited. Am J Physiol Endocrinol Metab 294:E882-888

8. Hajduch E, Litherland GJ, Hundal HS (2001) Protein kinase B (PKB/Akt) - a key regulator of glucose transport? FEBS Lett 492:199-203

9. Whiteman EL, Cho H, Birnbaum MJ (2002) Role of Akt/protein kinase B in metabolism. Trends Endocrinol Metab 13:444-451

10. Roden M, Price TB, Perseghin G et al (1996) Mechanism of free fatty acid-induced insulin resistance in humans. J Clin Invest 97:2859-2865

11. Roden M, Krssak M, Stingl H et al (1999) Rapid impairment of skeletal muscle glucose transport/phosphorylation by free fatty acids in humans. Diabetes 48:358-364

12. Vanhaesebroeck B, Alessi DR (2000) The PI3K-PDK1 connection: more than just a road to PKB. Biochem J 346(Pt 3):561-576

13. Milburn CC, Deak M, Kelly SM, Price NC, Alessi DR, Van Aalten DM (2003) Binding of phosphatidylinositol 3,4,5-trisphosphate to the pleckstrin homology domain of protein kinase B induces a conformational change. Biochem J 375:531-538

14. Liao Y, Hung MC (2010) Physiological regulation of Akt activity and stability. Am J Transl Res 2:19-42 
15. Chavez JA, Knotts TA, Wang LP et al (2003) A role for ceramide, but not diacylglycerol, in the antagonism of insulin signal transduction by saturated fatty acids. J Biol Chem 278:10297-10303

16. Holland WL, Brozinick JT, Wang LP et al (2007) Inhibition of ceramide synthesis ameliorates glucocorticoid-, saturatedfat-, and obesity-induced insulin resistance. Cell Metab 5:167-179

17. Schmitz-Peiffer C, Craig DL, Biden TJ (1999) Ceramide generation is sufficient to account for the inhibition of the insulin-stimulated PKB pathway in $\mathrm{C} 2 \mathrm{C} 12$ skeletal muscle cells pretreated with palmitate. J Biol Chem 274:24202-24210

18. Teruel T, Hernandez R, Lorenzo M (2001) Ceramide mediates insulin resistance by tumor necrosis factor- $\alpha$ in brown adipocytes by maintaining Akt in an inactive dephosphorylated state. Diabetes 50:2563-2571

19. Hajduch E, Balendran A, Batty IH et al (2001) Ceramide impairs the insulin-dependent membrane recruitment of protein kinase $\mathrm{B}$ leading to a loss in downstream signalling in L6 skeletal muscle cells. Diabetologia 44:173-183

20. Stratford S, Hoehn KL, Liu F, Summers SA (2004) Regulation of insulin action by ceramide: dual mechanisms linking ceramide accumulation to the inhibition of Akt/protein kinase B. J Biol Chem 279:36608-36615

21. Colombini M (2010) Ceramide channels and their role in mitochondria-mediated apoptosis. Biochim Biophys Acta 1797:1239-1244

22. Kolesnick RN, Kronke M (1998) Regulation of ceramide production and apoptosis. Annu Rev Physiol 60:643-665

23. Yang G, Badeanlou L, Bielawski J, Roberts AJ, Hannun YA, Samad F (2009) Central role of ceramide biosynthesis in body weight regulation, energy metabolism, and the metabolic syndrome. Am J Physiol Endocrinol Metab 297:E211-E224

24. Stancevic B, Kolesnick R (2010) Ceramide-rich platforms in transmembrane signaling. FEBS Lett 584:1728-1740

25. Won JS, Im YB, Khan M, Singh AK, Singh I (2004) The role of neutral sphingomyelinase produced ceramide in lipopolysaccharidemediated expression of inducible nitric oxide synthase. J Neurochem 88:583-593

26. Merrill AH Jr (2002) De novo sphingolipid biosynthesis: a necessary, but dangerous, pathway. J Biol Chem 277:25843-25846

27. Bose R, Verheij M, Haimovitz-Friedman A, Scotto K, Fuks Z, Kolesnick R (1995) Ceramide synthase mediates daunorubicininduced apoptosis: an alternative mechanism for generating death signals. Cell 82:405-414

28. Liao WC, Haimovitz-Friedman A, Persaud RS et al (1999) Ataxia telangiectasia-mutated gene product inhibits DNA damage-induced apoptosis via ceramide synthase. J Biol Chem 274:17908-17917

29. Watson ML, Coghlan M, Hundal HS (2009) Modulating serine palmitoyl transferase (SPT) expression and activity unveils a crucial role in lipid-induced insulin resistance in rat skeletal muscle cells. Biochem J 417:791-801

30. Liu YY, Han TY, Giuliano AE, Hansen N, Cabot MC (2000) Uncoupling ceramide glycosylation by transfection of glucosylceramide synthase antisense reverses adriamycin resistance. J Biol Chem 275:7138-7143

31. Granado MH, Gangoiti P, Ouro A, Arana L, Gomez-Munoz A (2009) Ceramide 1-phosphate inhibits serine palmitoyltransferase and blocks apoptosis in alveolar macrophages. Biochim Biophys Acta 1791:263-272

32. Virkamaki A, Korsheninnikova E, Seppala-Lindroos A et al (2001) Intramyocellular lipid is associated with resistance to in vivo insulin actions on glucose uptake, antilipolysis, and early insulin signaling pathways in human skeletal muscle. Diabetes 50:2337-2343
33. Bruce CR, Kriketos AD, Cooney GJ, Hawley JA (2004) Disassociation of muscle triglyceride content and insulin sensitivity after exercise training in patients with type 2 diabetes. Diabetologia 47:23-30

34. Summers SA, Garza LA, Zhou H, Birnbaum MJ (1998) Regulation of insulin-stimulated glucose transporter GLUT4 translocation and Akt kinase activity by ceramide. Mol Cell Biol 18:5457-5464

35. Powell DJ, Turban S, Gray A, Hajduch E, Hundal HS (2004) Intracellular ceramide synthesis and protein kinase $C \zeta$ activation play an essential role in palmitate-induced insulin resistance in rat L6 skeletal muscle cells. Biochem J 382:619-629

36. Chavez JA, Summers SA (2003) Characterizing the effects of saturated fatty acids on insulin signaling and ceramide and diacylglycerol accumulation in 3T3-L1 adipocytes and $\mathrm{C} 2 \mathrm{C} 12$ myotubes. Arch Biochem Biophys 419:101-109

37. Pickersgill L, Litherland GJ, Greenberg AS, Walker M, Yeaman SJ (2007) Key role for ceramides in mediating insulin resistance in human muscle cells. J Biol Chem 282:12583-12589

38. Samad F, Hester KD, Yang G, Hannun YA, Bielawski J (2006) Altered adipose and plasma sphingolipid metabolism in obesity: a potential mechanism for cardiovascular and metabolic risk. Diabetes 55:2579-2587

39. Blachnio-Zabielska A, Baranowski M, Zabielski P, Gorski J (2010) Effect of high fat diet enriched with unsaturated and diet rich in saturated fatty acids on sphingolipid metabolism in rat skeletal muscle. J Cell Physiol 225:786-791

40. Adams JM 2nd, Pratipanawatr T, Berria R et al (2004) Ceramide content is increased in skeletal muscle from obese insulinresistant humans. Diabetes 53:25-31

41. Straczkowski M, Kowalska I, Baranowski M et al (2007) Increased skeletal muscle ceramide level in men at risk of developing type 2 diabetes. Diabetologia 50:2366-2373

42. Turinsky J, Bayly BP, O'Sullivan DM (1990) 1,2-Diacylglycerol and ceramide levels in rat skeletal muscle and liver in vivo. Studies with insulin, exercise, muscle denervation, and vasopressin. J Biol Chem 265:7933-7938

43. Kim JK, Fillmore JJ, Chen Y et al (2001) Tissue-specific overexpression of lipoprotein lipase causes tissue-specific insulin resistance. Proc Natl Acad Sci USA 98:7522-7527

44. Bruce CR, Thrush AB, Mertz VA et al (2006) Endurance training in obese humans improves glucose tolerance and mitochondrial fatty acid oxidation and alters muscle lipid content. Am J Physiol Endocrinol Metab 291:E99-E107

45. Stratford S, DeWald DB, Summers SA (2001) Ceramide dissociates 3 '-phosphoinositide production from pleckstrin homology domain translocation. Biochem J 354:359-368

46. Cazzolli R, Carpenter L, Biden TJ, Schmitz-Peiffer C (2001) A role for protein phosphatase 2A-like activity, but not atypical protein kinase $\mathrm{C} \zeta$, in the inhibition of protein kinase B/Akt and glycogen synthesis by palmitate. Diabetes 50:2210-2218

47. Chalfant CE, Kishikawa K, Mumby MC, Kamibayashi C, Bielawska A, Hannun YA (1999) Long chain ceramides activate protein phosphatase-1 and protein phosphatase-2A. Activation is stereospecific and regulated by phosphatidic acid. J Biol Chem 274:20313-20317

48. Blouin CM, Prado C, Takane KK et al (2010) Plasma membrane subdomain compartmentalization contributes to distinct mechanisms of ceramide action on insulin signaling. Diabetes 59:600-610

49. Cortright RN, Azevedo JL Jr, Zhou Q et al (2000) Protein kinase $\mathrm{C}$ modulates insulin action in human skeletal muscle. Am J Physiol Endocrinol Metab 278:E553-E562

50. Bourbon NA, Sandirasegarane L, Kester M (2002) Ceramideinduced inhibition of Akt is mediated through protein kinase $\mathrm{C} \zeta$ : implications for growth arrest. J Biol Chem 277:3286-3292 
51. Powell DJ, Hajduch E, Kular G, Hundal HS (2003) Ceramide disables 3-phosphoinositide binding to the pleckstrin homology domain of protein kinase B (PKB)/Akt by a PKC $\zeta$-dependent mechanism. Mol Cell Biol 23:7794-7808

52. Stretton C, Evans A, Hundal HS (2010) Cellular depletion of atypical $\mathrm{PKC} \lambda$ is associated with enhanced insulin-sensitivity and glucose uptake in L6 rat skeletal muscle cells. Am J Physiol Endocrinol Metab 299:E402-E412

53. Hajduch E, Turban S, Le Liepvre X et al (2008) Targeting of $\mathrm{PKC} \zeta$ and $\mathrm{PKB}$ to caveolin-enriched microdomains represents a crucial step underpinning the disruption in PKB-directed signalling by ceramide. Biochem J 410:369-379

54. Doornbos RP, Theelen M, van der Hoeven PC, van Blitterswijk WJ, Verkleij AJ, van Bergen en Henegouwen PM (1999) Protein kinase $\mathrm{C} \zeta$ is a negative regulator of protein kinase B activity. J Biol Chem 274:8589-8596

55. Liu J, Schnitzer JE (1999) Analysis of lipids in caveolae. Meth Mol Biol 116:61-72

56. Simons K, Ikonen E (1997) Functional rafts in cell membranes. Nature 387:569-572

57. Chidlow JH Jr, Sessa WC (2010) Caveolae, caveolins, and cavins: complex control of cellular signalling and inflammation. Cardiovasc Res 86:219-225

58. Pilch PF, Souto RP, Liu L et al (2007) Cellular spelunking: exploring adipocyte caveolae. J Lipid Res 48:2103-2111

59. Oka N, Yamamoto M, Schwencke C et al (1997) Caveolin interaction with protein kinase $\mathrm{C}$. Isoenzyme-dependent regulation of kinase activity by the caveolin scaffolding domain peptide. J Biol Chem 272:33416-33421

60. Goswami R, Singh D, Phillips G, Kilkus J, Dawson G (2005) Ceramide regulation of the tumor suppressor phosphatase PTEN in rafts isolated from neurotumor cell lines. J Neurosci Res 81:541-550

61. Li L, Ren CH, Tahir SA, Ren C, Thompson TC (2003) Caveolin1 maintains activated Akt in prostate cancer cells through scaffolding domain binding site interactions with and inhibition of serine/threonine protein phosphatases PP1 and PP2A. Mol Cell Biol 23:9389-9404

62. Le Lay S, Kurzchalia TV (2005) Getting rid of caveolins: phenotypes of caveolin-deficient animals. Biochim Biophys Acta 1746:322-333

63. Simard JR, Meshulam T, Pillai BK et al (2010) Caveolins sequester FA on the cytoplasmic leaflet of the plasma membrane, augment triglyceride formation, and protect cells from lipotoxicity. J Lipid Res 51:914-922

64. Hanada K (2003) Serine palmitoyltransferase, a key enzyme of sphingolipid metabolism. Biochim Biophys Acta 1632:16-30

65. Ussher JR, Koves TR, Cadete VJ et al (2010) Inhibition of de novo ceramide synthesis reverses diet-induced insulin resistance and enhances whole body oxygen consumption. Diabetes 59:2453-2464

66. Gual P, Le Marchand-Brustel Y, Tanti JF (2005) Positive and negative regulation of insulin signaling through IRS-1 phosphorylation. Biochimie 87:99-109

67. Li Y, Soos TJ, Li X et al (2004) Protein kinase C $\theta$ inhibits insulin signaling by phosphorylating IRS1 at $\operatorname{Ser}^{1101}$. J Biol Chem 279:45304-45307

68. Pinnamaneni SK, Southgate RJ, Febbraio MA, Watt MJ (2006) Stearoyl CoA desaturase 1 is elevated in obesity but protects against fatty acid-induced skeletal muscle insulin resistance in vitro. Diabetologia 49:3027-3037

69. Holland WL, Miller RA, Wang ZV et al (2011) Receptormediated activation of ceramidase activity initiates the pleiotropic actions of adiponectin. Nat Med 17:55-63

70. Dobrzyn A, Zendzian-Piotrowska M, Gorski J (2004) Effect of endurance training on the sphingomyelin-signalling pathway activity in the skeletal muscles of the rat. J Physiol Pharmacol 55:305-313

71. Bikman BT, Zheng D, Reed MA, Hickner RC, Houmard JA, Dohm GL (2010) Lipid-induced insulin resistance is prevented in lean and obese myotubes by AICAR treatment. Am J Physiol Regul Integr Comp Physiol 298:R1692-1699

72. Blazquez C, Geelen MJ, Velasco G, Guzman M (2001) The AMP-activated protein kinase prevents ceramide synthesis de novo and apoptosis in astrocytes. FEBS Lett 489:149-153

73. Sriwijitkamol A, Coletta DK, Wajcberg E et al (2007) Effect of acute exercise on AMPK signaling in skeletal muscle of subjects with type 2 diabetes: a time-course and dose-response study. Diabetes 56:836-848

74. Merrill GF, Kurth EJ, Hardie DG, Winder WW (1997) AICA riboside increases AMP-activated protein kinase, fatty acid oxidation, and glucose uptake in rat muscle. Am J Physiol 273: E1107-E1112

75. Henique C, Mansouri A, Fumey G et al (2010) Increased mitochondrial fatty acid oxidation is sufficient to protect skeletal muscle cells from palmitate-induced apoptosis. J Biol Chem 285:36818-36827

76. Bruce CR, Hoy AJ, Turner N et al (2009) Overexpression of carnitine palmitoyltransferase- 1 in skeletal muscle is sufficient to enhance fatty acid oxidation and improve high-fat diet-induced insulin resistance. Diabetes 58:550-558

77. Coroneos E, Martinez M, McKenna S, Kester M (1995) Differential regulation of sphingomyelinase and ceramidase activities by growth factors and cytokines. Implications for cellular proliferation and differentiation. J Biol Chem 270:23305-23309

78. Boini KM, Zhang C, Xia M, Poklis JL, Li PL (2010) Role of sphingolipid mediator ceramide in obesity and renal injury in mice fed a high fat diet. J Pharmacol Exp Ther 334:839-846

79. Chocian G, Chabowski A, Zendzian-Piotrowska M, Harasim E, Lukaszuk B, Gorski J (2010) High fat diet induces ceramide and sphingomyelin formation in rat's liver nuclei. Mol Cell Biochem 340:125-131

80. Ferreira LF, Moylan JS, Gilliam LA, Smith JD, NikolovaKarakashian M, Reid MB (2010) Sphingomyelinase stimulates oxidant signaling to weaken skeletal muscle and promote fatigue. Am J Physiol Cell Physiol 299:C552-C560

81. Wong ML, Xie B, Beatini N et al (2000) Acute systemic inflammation up-regulates secretory sphingomyelinase in vivo: a possible link between inflammatory cytokines and atherogenesis. Proc Natl Acad Sci USA 97:8681-8686

82. Deevska GM, Rozenova KA, Giltiay NV et al (2009) Acid sphingomyelinase deficiency prevents diet-induced hepatic triacylglycerol accumulation and hyperglycemia in mice. J Biol Chem 284:8359-8368

83. Takeda K, Akira S (2005) Toll-like receptors in innate immunity. Int Immunol 17:1-14

84. Tsukumo DM, Carvalho-Filho MA, Carvalheira JB et al (2007) Loss-of-function mutation in Toll-like receptor 4 prevents dietinduced obesity and insulin resistance. Diabetes 56:1986-1998

85. Davis JE, Gabler NK, Walker-Daniels J, Spurlock ME (2008) Tlr-4 deficiency selectively protects against obesity induced by diets high in saturated fat. Obes Silver Spring 16:1248-1255

86. Memon RA, Holleran WM, Moser AH et al (1998) Endotoxin and cytokines increase hepatic sphingolipid biosynthesis and produce lipoproteins enriched in ceramides and sphingomyelin. Arterioscler Thromb Vasc Biol 18:1257-1265

87. Langeveld M, Ghauharali KJ, Sauerwein HP et al (2008) Type I Gaucher disease, a glycosphingolipid storage disorder, is associated with insulin resistance. J Clin Endocrinol Metab 93:845-851

88. Tagami S, Inokuchi Ji J, Kabayama K et al (2002) Ganglioside GM3 participates in the pathological conditions of insulin resistance. J Biol Chem 277:3085-3092 
89. Kabayama K, Sato T, Kitamura F et al (2005) TNF $\alpha$-induced insulin resistance in adipocytes as a membrane microdomain disorder: involvement of ganglioside GM3. Glycobiology 15:21-29

90. Aerts JM, Ottenhoff R, Powlson AS et al (2007) Pharmacological inhibition of glucosylceramide synthase enhances insulin sensitivity. Diabetes 56:1341-1349

91. Yamashita T, Hashiramoto A, Haluzik M et al (2003) Enhanced insulin sensitivity in mice lacking ganglioside GM3. Proc Natl Acad Sci USA 100:3445-3449

92. Zhao H, Przybylska M, Wu IH et al (2007) Inhibiting glycosphingolipid synthesis improves glycemic control and insulin sensitivity in animal models of type 2 diabetes. Diabetes 56:1210-1218

93. Kabayama K, Sato T, Saito K et al (2007) Dissociation of the insulin receptor and caveolin-1 complex by ganglioside GM3 in the state of insulin resistance. Proc Natl Acad Sci USA 104:13678-13683

94. Yoshizumi S, Suzuki S, Hirai M et al (2007) Increased hepatic expression of ganglioside-specific sialidase, NEU3, improves insulin sensitivity and glucose tolerance in mice. Metabolism $56: 420-429$
95. Sato T, Nihei Y, Nagafuku M et al (2008) Circulating levels of ganglioside GM3 in metabolic syndrome: a pilot study. Obes Res Clin Pract 2:231-238

96. Yu C, Chen Y, Cline GW et al (2002) Mechanism by which fatty acids inhibit insulin activation of insulin receptor substrate-1 (IRS-1)-associated phosphatidylinositol 3-kinase activity in muscle. J Biol Chem 277:50230-50236

97. Skovbro M, Baranowski M, Skov-Jensen C et al (2008) Human skeletal muscle ceramide content is not a major factor in muscle insulin sensitivity. Diabetologia 51:1253-1260

98. Serlie MJ, Meijer AJ, Groener JE et al (2007) Short-term manipulation of plasma free fatty acids does not change skeletal muscle concentrations of ceramide and glucosylceramide in lean and overweight subjects. J Clin Endocrinol Metab 92:1524-1529

99. Minehira K, Young SG, Villanueva CJ et al (2008) Blocking VLDL secretion causes hepatic steatosis but does not affect peripheral lipid stores or insulin sensitivity in mice. J Lipid Res 49:2038-2044

100. Pavoine C, Pecker F (2009) Sphingomyelinases: their regulation and roles in cardiovascular pathophysiology. Cardiovasc Res $82: 175-183$ 